\title{
The Unified Process for Component-Based Development
}

\author{
Ivar Jacobsen \\ Rational Software Corporation \\ ivar@Rational.Com
}

\begin{abstract}
A better development process-in fact, a process unifying the best practices now available-is the key to the software future. The proven Unified Process originally developed by Ivar Jacobson, now incorporating the work of Grady Booch, Jim Rumbaugh, Philippe Kruchten, Walker Royce, and other people inside Rational, answers this long-felt need.

Component and object based, the Unified Process enables reuse. Usecase driven, it closes the gap between what the user needs and what the developer does, it drives the development process. Architecture centric, it guides the development process. Iterative and incremental, it manages risk. Represented in the design blueprints of the newly standardized Unified Modeling Language (UML), it communicates your results to a wide audience.
\end{abstract}

